\title{
Pulse line ion accelerator based design for the neutralized drift chamber experiment
}

\author{
C. Y. Ling, ${ }^{1, *}$ S. S. Yu, ${ }^{1,2}$ E. Henestroza, ${ }^{2}$ D. P. Grote, ${ }^{3}$ and A. Friedman ${ }^{3}$ \\ ${ }^{1}$ The Chinese University of Hong Kong, Hong Kong \\ ${ }^{2}$ Lawrence Berkeley National Laboratory, Berkeley, California 94720, USA \\ ${ }^{3}$ Lawrence Livermore National Laboratory, Livermore, California 94550, USA
}

(Received 5 November 2008; published 2 April 2009)

\begin{abstract}
The pulse line ion accelerator (PLIA) is a promising approach to accelerating heavy ion beams to regimes of interest for the study of high energy density physics (HEDP) and warm dense matter in a cost effective way. In this paper we demonstrate a PLIA-based design for a proposed HEDP machine, the neutralized drift compression experiment II. The simulation results from the injector to the exit of the accelerator using the particle-in-cell code WARP are presented. We show how the traveling wave structure of PLIA suggests straightforward strategies in controlling the longitudinal and transverse dynamics of the ion beam.
\end{abstract}

DOI: 10.1103/PhysRevSTAB.12.040401

\section{INTRODUCTION}

The pulse line ion accelerator (PLIA) [1] provides a lowcost approach to accelerating well-controlled heavy ion beams to regimes of interest for the study of high energy density physics and warm dense matter [2,3]. A voltage pulse is applied to the front end of the PLIA and generates a traveling electromagnetic wave pulse moving down a helical structure. The ion beam can be accelerated and longitudinally confined by a judicious choice of waveforms and wave velocities [4]. The wave velocity $v_{w}$ is determined by the impedance $L_{0}$ and the capacitance $C_{0}$ of the helix. Proof-of-principle experiments had been performed at Lawrence Berkeley National Laboratory (LBNL) [5].

The neutralized drift compression experiment II (NDCX-II) is being pursued by the Heavy Ion Fusion Science Virtual National Laboratory, a collaboration of LBNL, Lawrence Livermore National Laboratory, and the Princeton Plasma Physics Laboratory [6,7]. The application is to heat a target by use of an ion beam to the regime of warm dense matter and high energy density physics [8]. A design example of interest is a lithium ion beam with energy $2.8 \mathrm{MeV}$, total charge $30 \mathrm{nC}$, bunch length $1 \mathrm{~ns}$, and spot size $<1 \mathrm{~mm}$ at target [9]. The PLIA concept is advantageous to acceleration of such an intense ion beam due to its traveling wave characteristics. Since the voltage pulse can be applied immediately after the entire ion beam enters the helical pulse line, the tail of the ion beam is accelerated by the voltage waveform at earlier time and the pulse sweeps through the ion beam. In this way the ion beam can be bunched. The structure of a PLIA is in several respects simple relative to other options $[3,6,9]$ and so it has the potential for low development cost. Those features

\footnotetext{
*cyling@cuhk.edu.hk
}

PACS numbers: 29.20.-c, 29.27.Ac, 29.27.Eg, 41.75.Ak

of PLIA make it an interesting alternative to the induction cell approach.

The proposed design for the PLIA-based accelerator for NDCX-II was simulated using the particle-in-cell code WARP [10]. An axisymmetric version, WARPRZ [11], was adopted for these studies. The simulation proceeds from the extraction of the particles at the source to the end of the accelerator. It includes longitudinal and transverse dynamics.

The paper is organized as follows: Section II introduces the basic strategy and principle adopted in the design. Section III demonstrates and discusses the simulation results for longitudinal and transverse dynamics, respectively. A conclusion is presented in Sec. IV, which also discusses the possible future development of the PLIA approach.

\section{BASIC DESIGN STRATEGY}

In the helix model employed in the simulations, $15 \mathrm{~cm}$ of resistive string is assumed at two ends of each helix and their resistances are assumed to be perfectly matched with the helix. Within the region of resistive string, a constant voltage gradient is assumed. The waveform moves down the helix from the beginning to the end with constant speed and without dispersion. Also the waveform is independent of the radial position inside the helix. This model has been checked against fully 3D electromagnetic calculations using the MAFIA code [12]. The model of space charge employed is fully electrostatic in the simulations. A comparison of simulation against experimental results is given in [4] — where the details of longitudinal phase space (which is quite complicated) have been reproduced. Exactly the same model used in [4] was applied in the present paper. The typical number of macroparticles is around $3 \times 10^{4}$, representing around $2 \times 10^{11}$ of real ion particles. The grid size is $4 \mathrm{~mm}$. Results are essentially the 

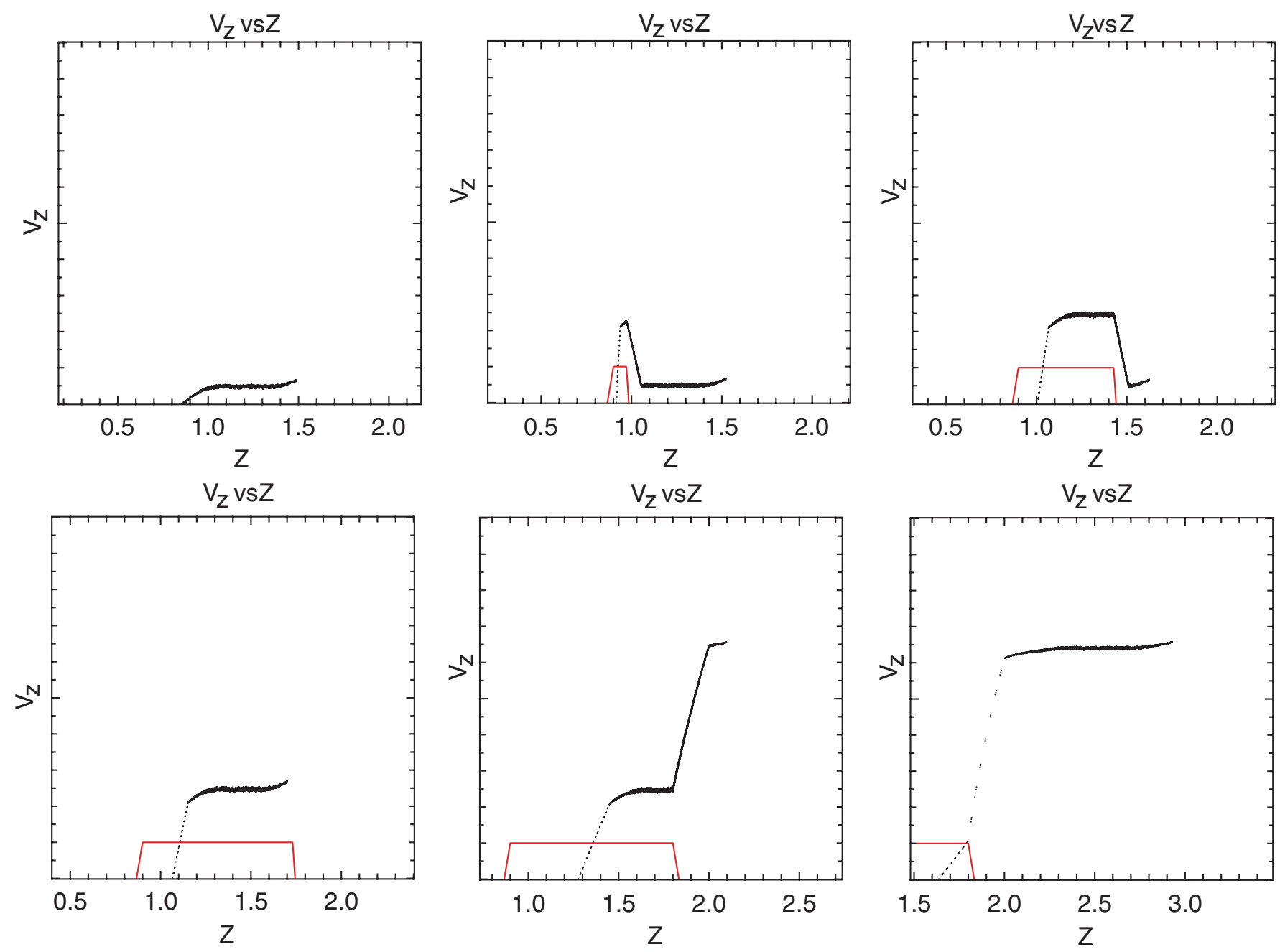

FIG. 1. (Color) Snapshots of the phase space of the ion beam (black) in the beam frame being accelerated by a voltage waveform (red) in snowplow mode. The helix starts at $z=0.7 \mathrm{~m}$ and ends at $z=1.9 \mathrm{~m}$. In the first four panels, the voltage wave sweeps through the ion beam and reaches the end of the helix. Then the ion beam gains an extra voltage $\Delta V$ when it exits the helix tube, as shown in the last two panels.

same when further increasing the number of grids and/or number of particles.

In the design concept presented here, the accelerator consists of several PLIA sections. In each section, the "snowplow mode" of acceleration was adopted. In this mode, the voltage waveform is applied at the input of the pulse line after the entire ion beam has entered within the helix. The wave speed $v_{w}$ is set to be higher than the initial ion beam velocity so that the voltage waveform sweeps through the entire ion beam before it reaches the end of the helix. The acceleration schedule is determined by the input voltage waveform $V(t)$ and $v_{w}$. In addition, the ion beam is bunched in this mode since the tail of the ion beam is accelerated first. Figure 1 shows the process of the ion beam accelerated by a single-ramped waveform in the snowplow mode.

The voltage waveform used in the design consists of two linear ramps, as shown in Fig. 2. The first ramp is shorter and steeper, which is mainly for acceleration. The second ramp cancels the space charge effect and hence suppresses the lengthening of the beam. Details of the design strategy are explained below.

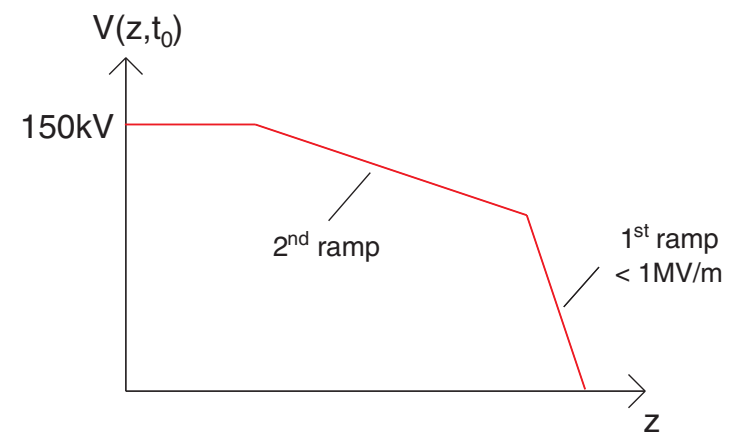

FIG. 2. (Color) A voltage waveform consists of two ramps. 

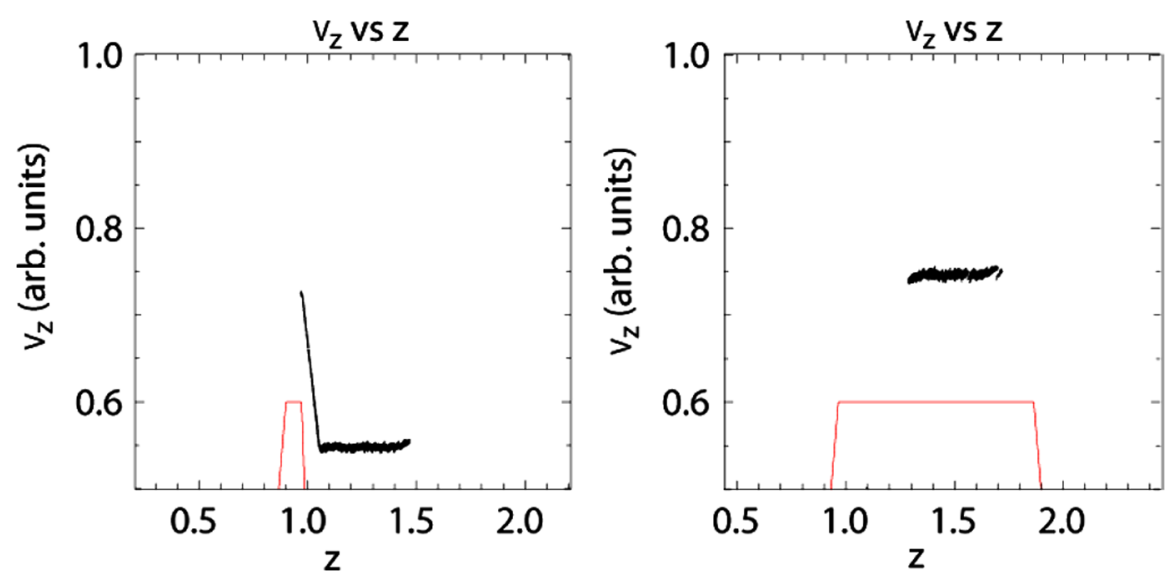

FIG. 3. (Color) The phase space of the ion beam is lifted horizontally after the voltage swept through it. The helix starts at $z=0.7 \mathrm{~m}$ and ends at $z=1.9 \mathrm{~m}$.

The acceleration by the first ramp can be understood by sweeping a low current beam (for which the space charge effect does not dominate) with a single short ramp. As can be seen from Fig. 3, the phase space of the ion beam is being horizontally lifted and the energy gain $\Delta E$ of each part of the beam is the same. In this case, $\Delta E$ is determined by the wave speed $v_{w}$ and the voltage $\Delta V$ of the ramp, provided that it is in snowplow mode. For a given voltage waveform, Fig. 4 shows the effect of different $v_{w}$ on energy gain per unit length, which is larger for lower $v_{w}$, due to the fact that any given ion has a longer time to stay in the region of accelerating gradient.

When the space charge effect of the ion beam is dominant, as is the case in our NDCX-II design, the distribution

\section{lower wave speed}
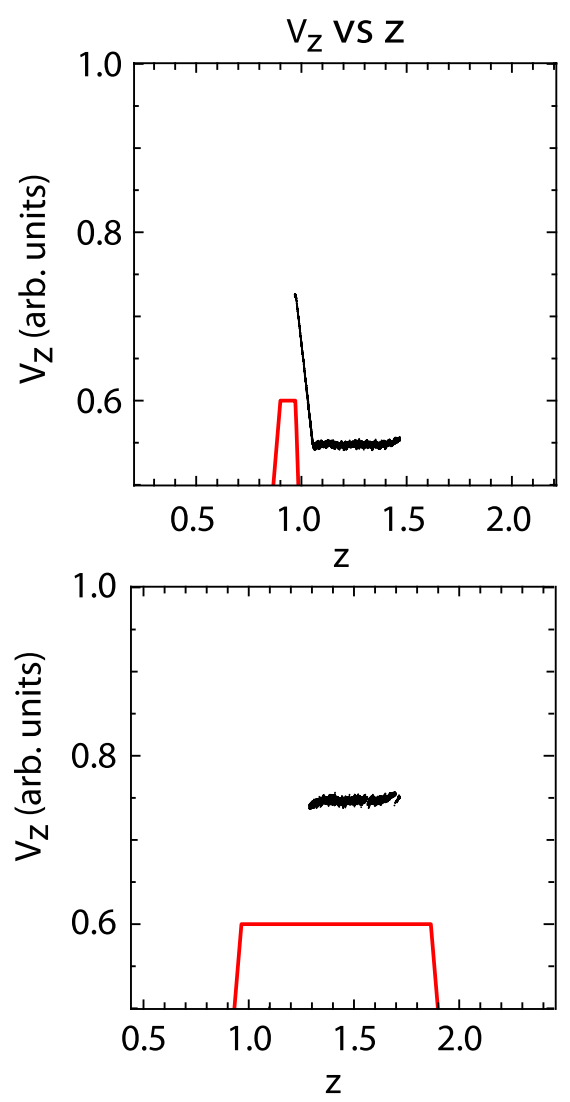

\section{higher wave speed}

$\mathrm{V}_{\mathrm{Z}}$ VS Z
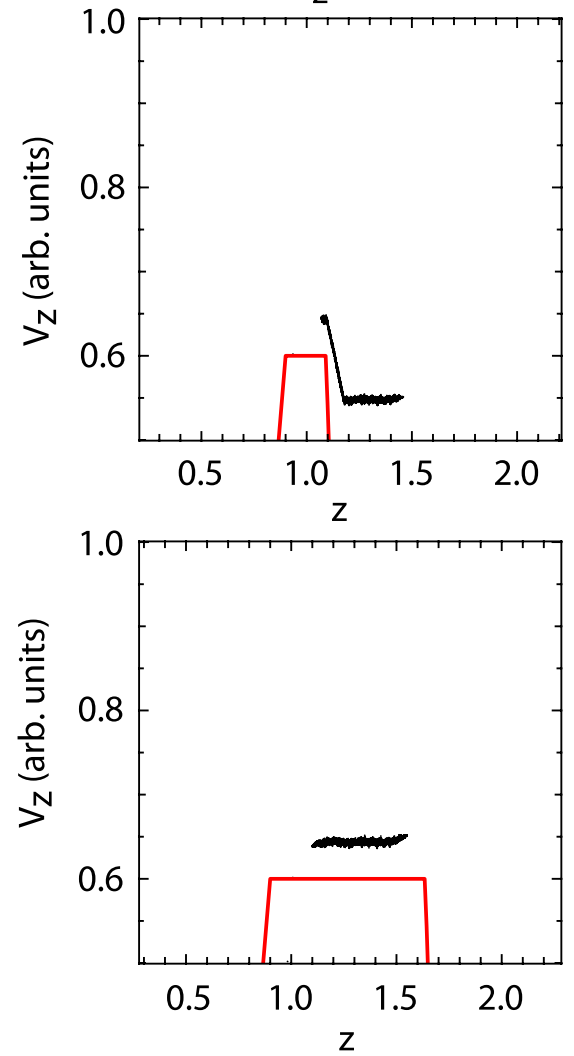

FIG. 4. (Color) Higher wave speed results in lower energy gain of the ion beam. Both helices start at $z=0.7 \mathrm{~m}$ and end at $z=1.9 \mathrm{~m}$. 

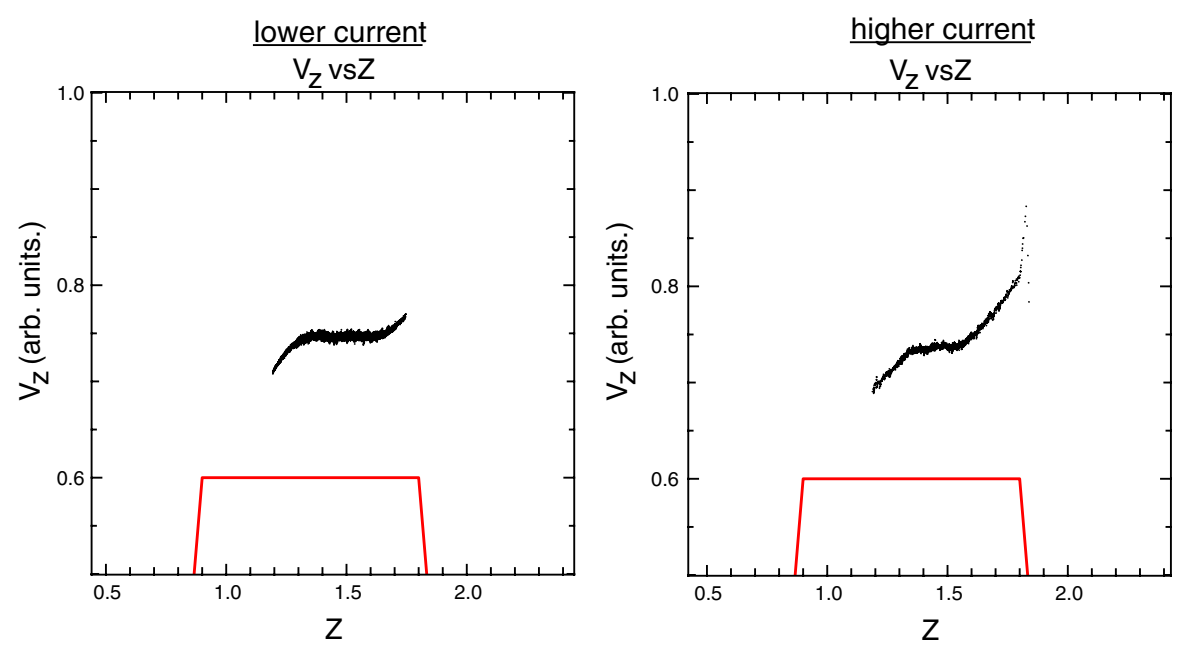

FIG. 5. (Color) The space charge force distorts the phase space of the ion beam. The helix starts at $z=0.7 \mathrm{~m}$ and ends at $z=1.9 \mathrm{~m}$.
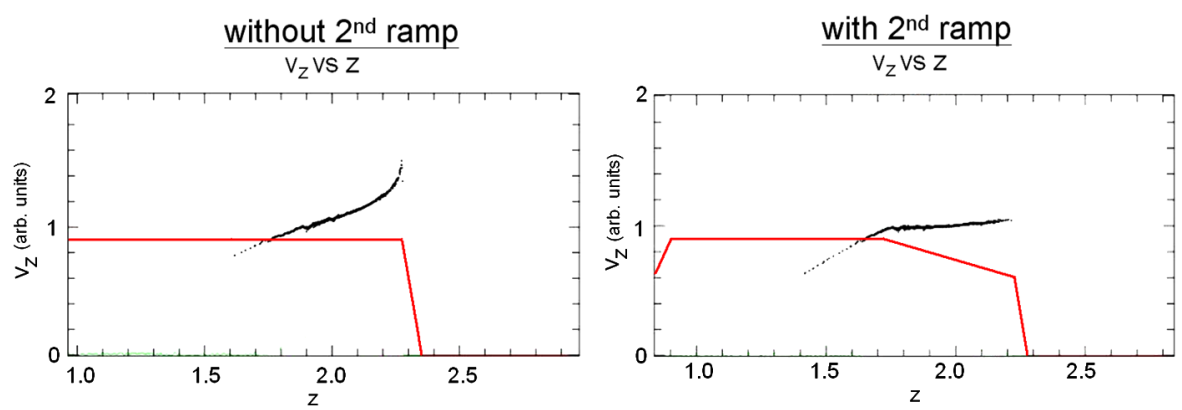

FIG. 6. (Color) The resultant phase space with and without the second ramp in the voltage waveform. Both helices start at $z=0.7 \mathrm{~m}$ and end at $z=1.9 \mathrm{~m}$.

of the ion beam in phase space becomes more difficult to control. The space charge force within the ion beam pushes ions towards the two ends and as a result the ion beam head has more energy than the tail. The slope of the phase space of the ion beam becomes positive and it lengthens. This is not desirable since a longer section of helices would then have to be used, the line charge density of the ion beam would be lowered and the velocity tilt (head-to-tail variation) would be difficult to control. Figure 5 compares the simulation of the phase space of the low current ion beam (negligible space charge) and the high current ion beam (space charge dominated). Instead of having the same energy everywhere in the ion beam, the higher current beam has a phase space with a positive slope. In order to keep the phase space "flat," more energy has to be given to the tail of the ion beam. Therefore, the second ramp is introduced to serve this purpose as shown in Fig. 6.

Now consider the idealized situation: the ion beam keeps the parabolic line charge density with the same beam length throughout the acceleration. If

$$
\lambda(s)=-\frac{3 Q}{4 l^{3}}(s-l)(s+l)
$$

where $Q$ is the total charge of the ion beam with length $2 l$ and $s$ is the longitudinal coordinate in the beam frame (see Fig. 7), then (in the long-wavelength limit) the electric field $E_{\mathrm{sc}}(s)$ due to space charge experienced by a slice of beam at $s$ is

$$
E_{\mathrm{sc}}(s)=-g \frac{d \lambda}{d s}=g \frac{3 Q}{2 l^{3}} s .
$$

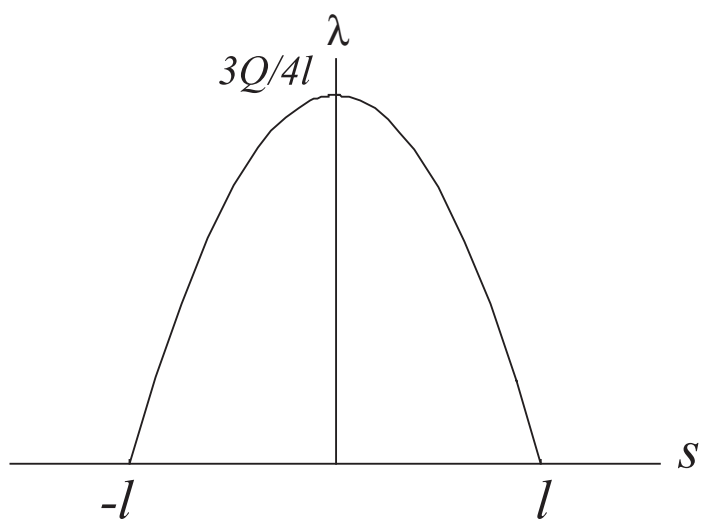

FIG. 7. Parabolic line charge density with ion beam length $2 l$. 
where $g$ is a dimensionless factor of order unity. The energy change $\Delta K_{\text {internal }}(s)$ of each particle of the ion beam after it drifts through a distance $d$ is

$$
\Delta K_{\text {internal }}(s)=\frac{3 q g Q d}{2 l^{3}} s,
$$

which is linear in $s$.

Assume that every slice of the beam has the same velocity $v_{\text {beam }}$. If the snowplow mode is used and the ramp is long enough so that the tail of the beam does not reach the flattop region of the voltage wave, the distance traveled by the slice of the ion beam when it is in the field region, $\tilde{d}(s)$, depends on the difference of the velocity of the wave $v_{w}$ and the ion beam $v_{\text {beam }}$, and is linear in $s$,

$$
\tilde{d}(s)=d_{0}+\frac{v_{\text {beam }}}{v_{w}-v_{\text {beam }}}(l-s) .
$$

Here $d_{0}$ is the distance traveled by the beam after the entire beam enters the field region. If a linear ramp is used, that is, the electric field $E_{z}$ is constant throughout the ramp, the energy gain of each ion particle of each slice of the beam

$$
\begin{aligned}
\Delta K_{\text {external }}(s) & =q E_{z} \tilde{d}(s) \\
& =q E_{z} d_{0}+q E_{z} \frac{v_{\text {beam }}}{\boldsymbol{v}_{w}-\boldsymbol{v}_{\text {beam }}}(l-s) .
\end{aligned}
$$

If the $v_{w}$ (or the length of the helical pulse line) is set such that the beam head exits at the same time that the entire beam has entered the field region, $d_{0}=0$. Then, the total energy change of the ion particle at $s$ is

$$
\begin{aligned}
\Delta K_{\text {total }}(s) & =\Delta K_{\text {internal }}(s)+\Delta K_{\text {external }}(s) \\
& =\frac{3 g q Q d}{2 l^{3}} s+q E_{z} \frac{v_{\text {beam }}}{v_{w}-v_{\text {beam }}}(l-s) .
\end{aligned}
$$

If we set

$$
E_{z}=\frac{3 g Q d}{2 l^{3}} \frac{v_{w}-v_{\text {beam }}}{v_{\text {beam }}},
$$

the energy change $\Delta K_{\text {total }}(s)$ of the particle is independent of $s$. The space charge effect can thus be compensated by applying the linear ramp in snowplow mode. This tworamp voltage waveform design is simple but effective for the PLIA-based NDCX-II.

The voltage limit $V_{\max }$ and the maximum gradient $E_{\max }$ was set (somewhat arbitrarily) to be $150 \mathrm{kV}$ and $1 \mathrm{MV} / \mathrm{m}$, respectively. The available parameters for the design of each helix are the time $t_{\text {start }}$ when the voltage wave is applied, the wave speed $v_{w}$, the length $d$, the voltage of the second ramp $V_{2}\left(V_{2}=V_{\max }-V_{1}\right.$, where $V_{1}$ is the voltage of the first ramp), the length of the first ramp $l_{r 1}$, and the length of the second ramp $l_{r 2}$. $t_{\text {start }}$ is set such that the voltage wave is applied once the entire ion beam has passed the first juncture of the resistive string and the helix wire. As discussed in the previous section, the energy gain $\Delta E$ is greater if $v_{w}$ is smaller. However, if $v_{w}$ is too small, the ion beam may be overbunched, the space charge effect would be extremely dominant, and hence the phase space would be seriously distorted. Furthermore, overtaking off slices within the beam may occur. Therefore, $v_{w}$ is optimized in this sense. It is also desired that the ion beam reaches the end of the helix after the first ramp has swept through the entire ion beam and the region of the second ramp has just reached the head of the ion beam so as to make $d_{0}=0 . d$ is set to achieve this. $l_{r 1}$ is equal to the shortest possible ramp length such that the gradient does not exceed $E_{\max }$, as mentioned in the previous section. $l_{r 2}$ should be long enough so that the tail of the ion beam does not reach the flattop region of the voltage waveform. Finally, except that the full voltage of the waveform does not exceed the limit $V_{\max }, V_{2}$ (and hence $V_{1}=V_{\max }-V_{2}$ ) is chosen to give a suitable acceleration field for suppression of the lengthening of the ion beam. However, in the last section of the helical pulse line, the velocity of the wave is chosen to be just a little bit higher than the initial velocity of the ion beam. It is to impose a velocity tilt to the ion beam before entering the induction section for voltage tailoring and the drift compression section. When the ion beam exits the helix, it gains an additional energy of $q V_{\max }$ (where $q$ is the charge of an ion) as shown in Fig. 1 (note that the ion beam does not have the same energy gain when it enters the helix because the voltage wave is always applied after the whole ion beam has entered the helical pulse line). The helix-to-helix distance is set to be $15 \mathrm{~cm}$ which allows for intercell vacuum pumping, diagnostics etc.

Besides the longitudinal dynamics, the envelope of the ion beam was also maintained within the pipe radius $7 \mathrm{~cm}$ by imposing an appropriate solenoidal field. In the injector section, there are three solenoids, which produces ion beam with radius $\sim 3 \mathrm{~cm}$. For each helix section, there is a solenoid surrounding it. In the present design, we allow

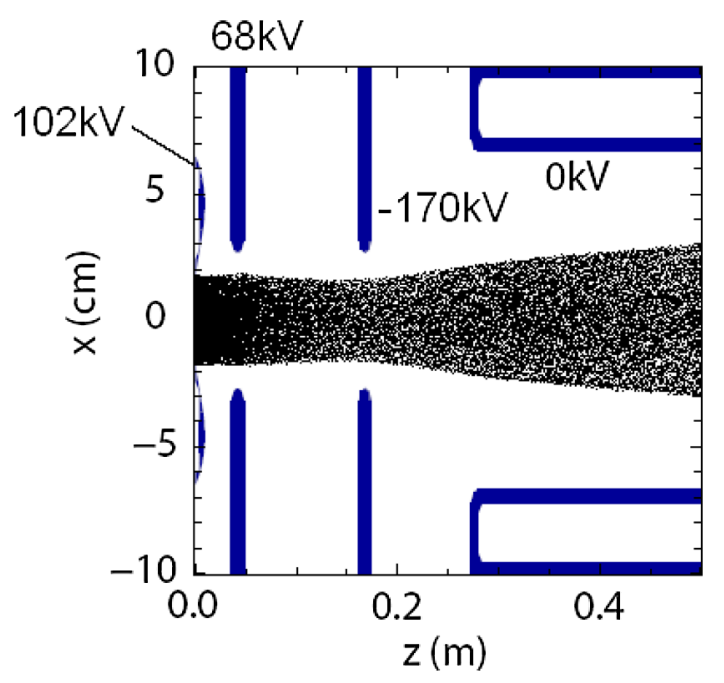

FIG. 8. (Color) The lithium diode injector and snapshot of the beam. 

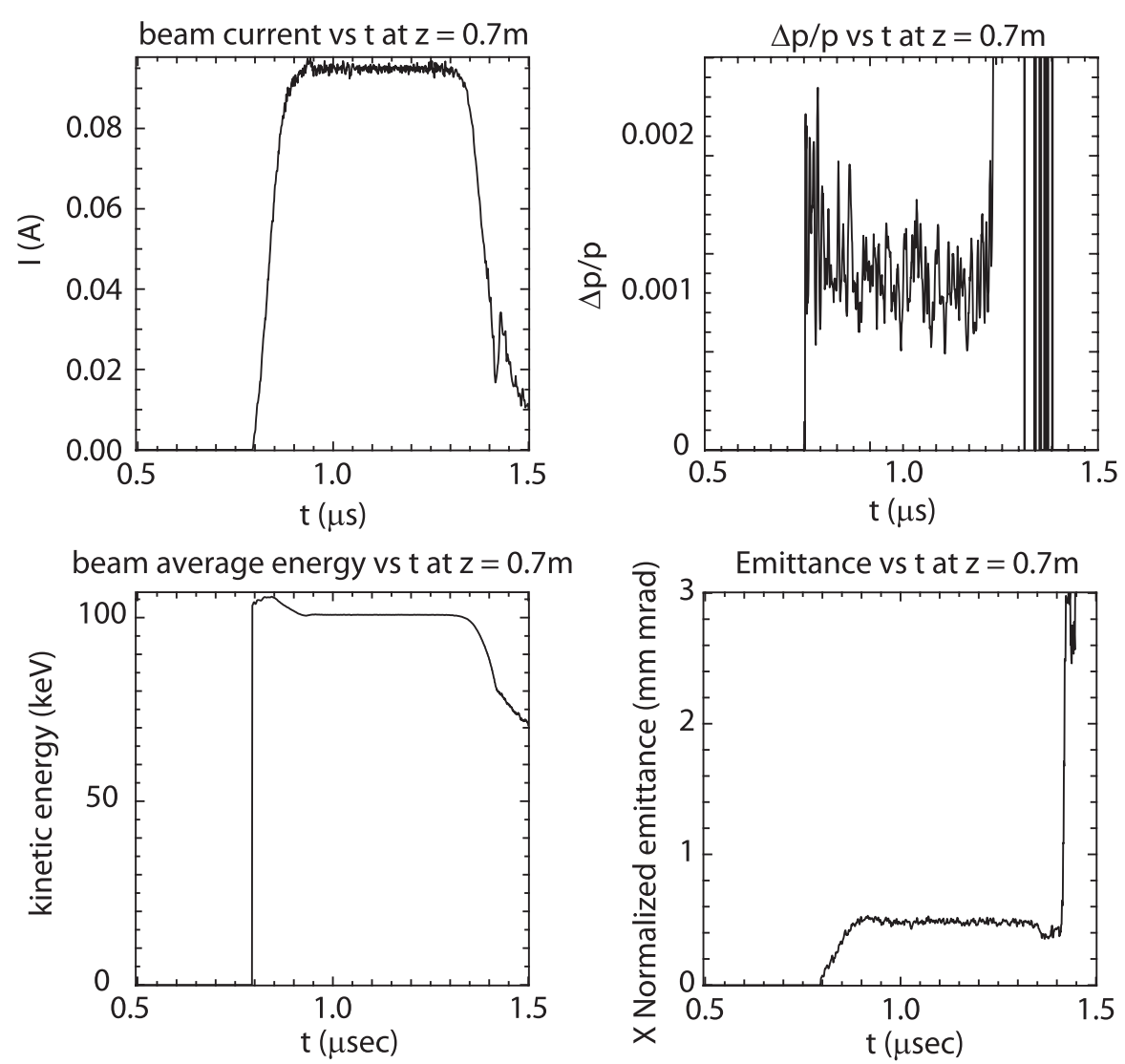

FIG. 9. (Color) The ion beam measured at the beginning of the first helical pulse line; the red part of the trace (in this and subsequent figures) indicates the region with current exceeding $80 \%$ of the peak current of the ion beam.

the separation between solenoids to be $15 \mathrm{~cm}$, again for engineering purposes. The field strength of each solenoid was designed to match the ion beam transversely for each helix section and to minimize emittance growth.

\section{SIMULATION RESULTS}

Following the design strategy described in the previous section, we obtained an optimized longitudinal design. The entire NDCX-II machine consists of a lithium injector, five sections of helical pulse line, the induction cells for voltage tailoring, and a drift compression section. We present simulation results obtained from WARP for the above design, up to the end of the last helical section. Figure 8 shows the lithium diode injector. It is composed of an extraction plate, accelerating region, and decelerating region. The potential of the extractor plate is set at $60 \mathrm{kV}$, followed by two metal plates which are set at 41.5 and $-100 \mathrm{kV}$, respectively. Then the ion beam enters the decelerating region which aims at compressing the beam longitudinally. The injector is $40 \mathrm{~cm}$ long. The injector design was simulated and the diagnostics of the ion beam at the exit of the injector are shown in Fig. 9.

Following the diode, there are five sections of helical pulse line, with the first one beginning at $z=70 \mathrm{~cm}$. The parameters of the helical section are listed in Table I. As mentioned before, snowplow mode and two-ramp voltage waveform were adopted for the five helical pulse lines. In the simulation, it is assumed that no dispersion occurs during the propagation of the voltage waveform and that it travels with constant speed $v_{w}$ along each helical line.

Figure 10 shows the phase space of the ion beam at different times throughout the five sections of the helical pulse line. From sections 1 to 4 , we have tried to follow a design strategy to maintain a nearly flat phase space at exits, but with a slightly positive gradient to minimize particle overtaking. In section 5 , we adjust the voltage waveform (with a single ramp) and the section length to achieve a negative slope for the longitudinal phase space at acceleration exit. This is in preparation for the subsequent

TABLE I. Parameters of each helix section.

\begin{tabular}{lcccccc}
\hline \hline Helix & $\begin{array}{c}d \\
(\mathrm{~m})\end{array}$ & $\begin{array}{c}v_{w} \\
(\mathrm{~m} / \mu \mathrm{s})\end{array}$ & $\begin{array}{c}l_{r 1} \\
(\mathrm{~m})\end{array}$ & $\begin{array}{c}V_{1} \\
(\mathrm{kV})\end{array}$ & $\begin{array}{c}l_{r 2} \\
(\mathrm{~m})\end{array}$ & $\begin{array}{c}V_{2} \\
(\mathrm{kV})\end{array}$ \\
\hline 1 & 2.25 & 3.8 & 0.12 & 120 & 0.85 & 30 \\
2 & 2.70 & 5.8 & 0.14 & 140 & 0.6 & 10 \\
3 & 3.15 & 7.5 & 0.1 & 100 & 0.6 & 50 \\
4 & 3.65 & 8.8 & 0.1 & 80 & 0.6 & 70 \\
5 & 4.35 & 9.5 & 0.55 & 150 & 0 & 0 \\
\hline \hline
\end{tabular}



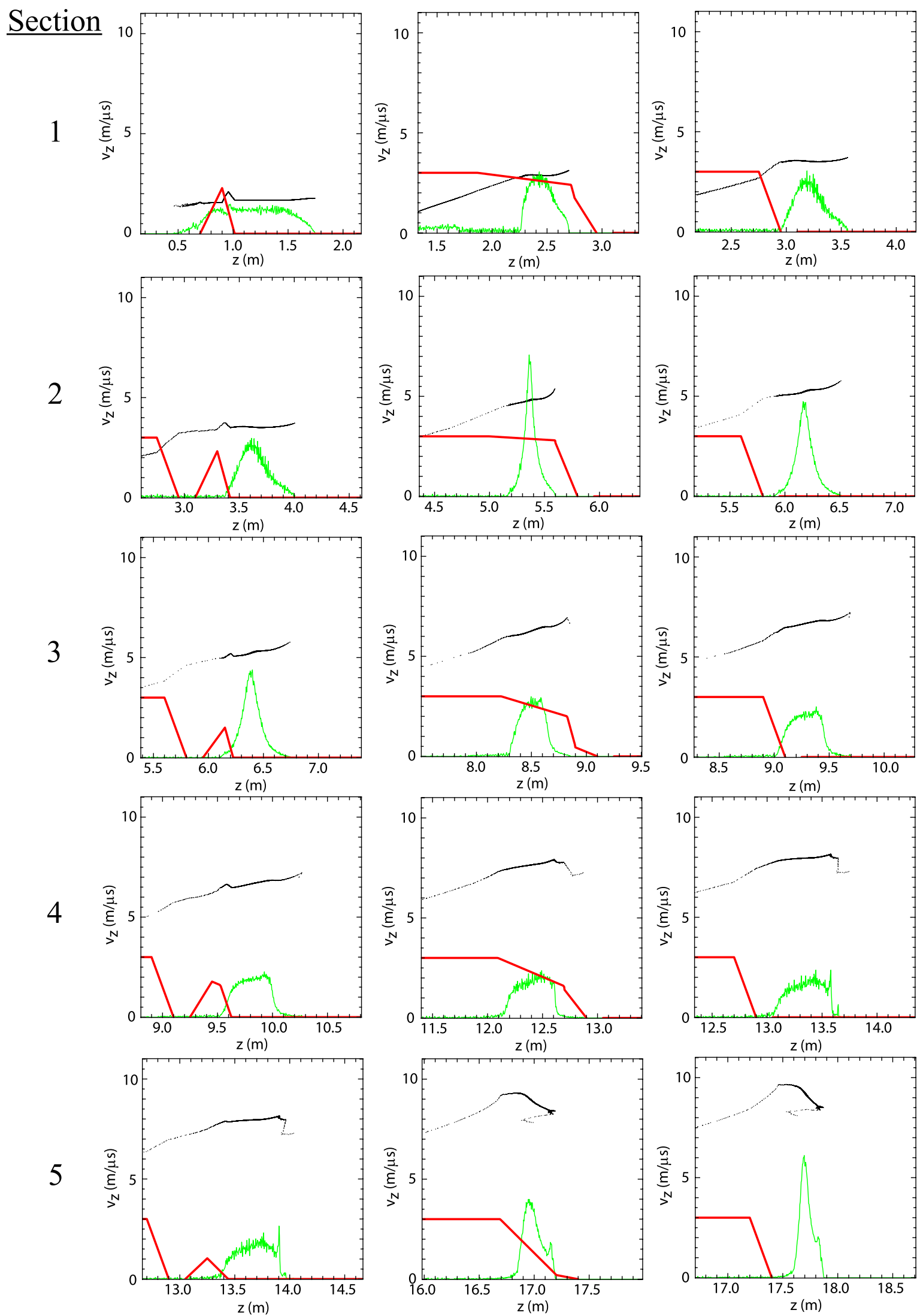

FIG. 10. (Color) The phase space of the ion beam when (i) it has entered the helix entirely, (ii) the voltage wave has swept through the entire ion beam, and (iii) the entire ion beam has exited the helix, for every section of the helix. 

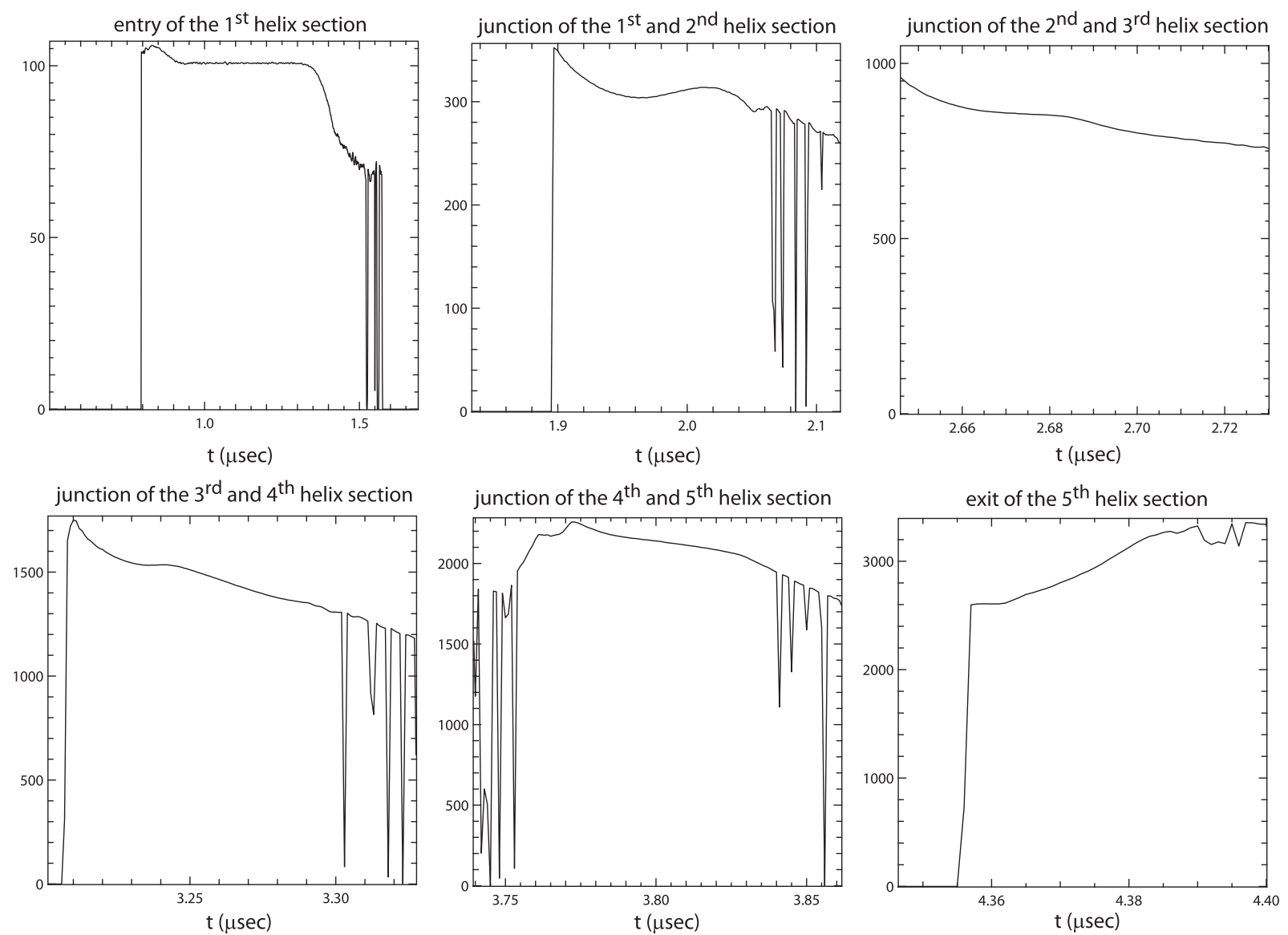

FIG. 11. The energy (in $\mathrm{keV}$ ) of the ion beam versus time at various positions outside the helices.
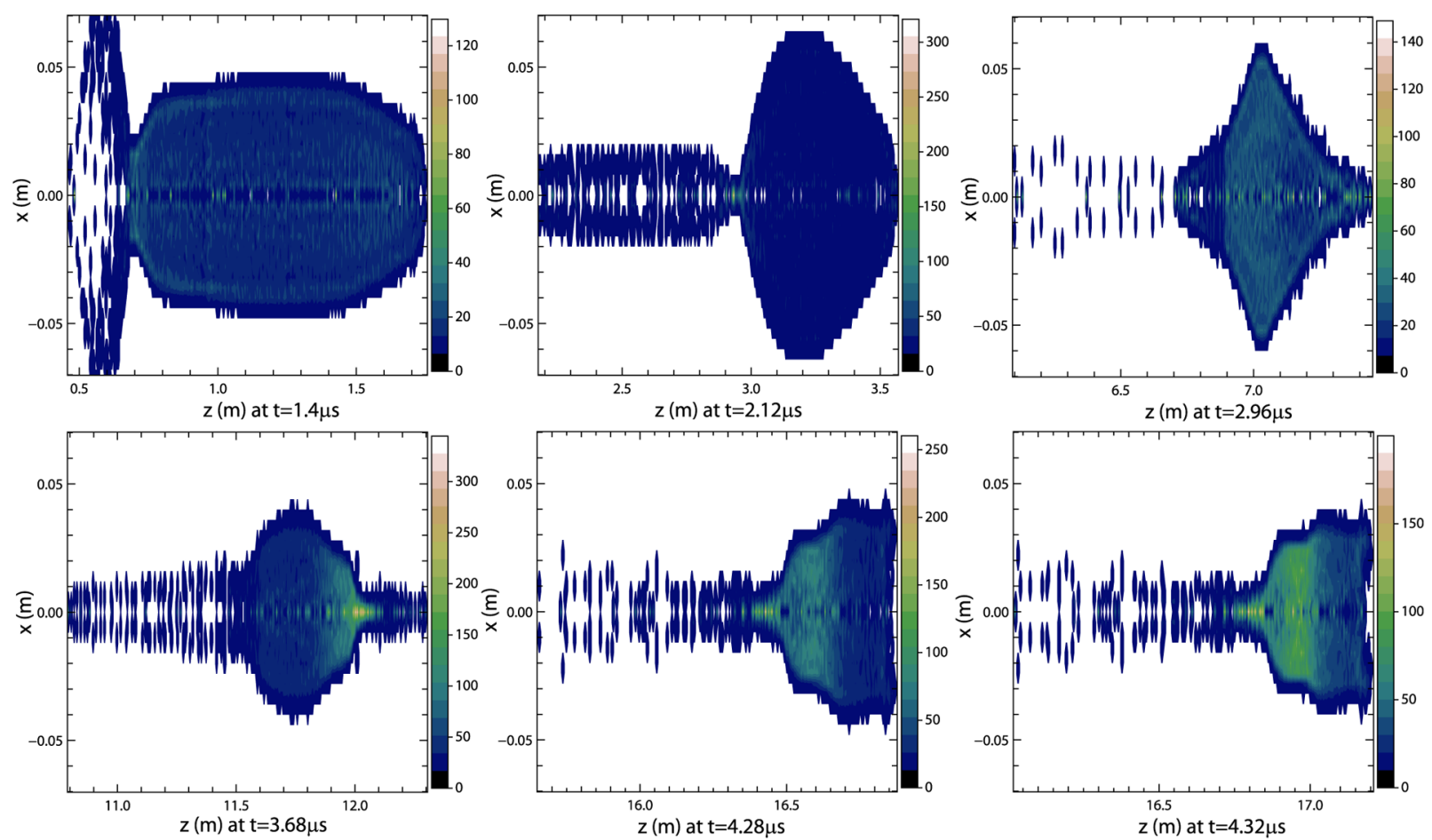

FIG. 12. (Color) Contours of charge density (in $\mu \mathrm{C} / \mathrm{m}^{3}$ ) of the ion beam in the $X-Z$ plane at different times. 
TABLE II. Positions and on-axis field of solenoid.

\begin{tabular}{|c|c|c|c|c|c|c|}
\hline Solenoid & Starting position $(\mathrm{m})$ & End position $(\mathrm{m})$ & Length $(\mathrm{m})$ & Inner radius $(\mathrm{cm})$ & Outer radius $(\mathrm{cm})$ & Field on axis (T) \\
\hline 1 & -0.015 & 0.015 & 0.03 & 12 & 13 & -0.0004 \\
\hline 2 & 0.404 & 0.504 & 0.10 & 7 & 8 & 0.76 \\
\hline 3 & 0.524 & 0.624 & 0.10 & 7 & 8 & 0.43 \\
\hline 4 & 0.7 & 2.92 & 2.22 & 7.7 & 9.7 & 0.29 \\
\hline 5 & 3.07 & 5.42 & 2.25 & 7.7 & 9.7 & 0.36 \\
\hline 6 & 5.57 & 8.13 & 2.56 & 7.7 & 9.7 & 0.43 \\
\hline 7 & 8.28 & 11.58 & 3.30 & 7.7 & 9.7 & 0.43 \\
\hline 8 & 11.73 & 18 & 6.27 & 7.7 & 9.7 & 0.36 \\
\hline
\end{tabular}

neutralized drift compression section. We note that using the design voltage waveforms, the exiting phase space indeed has the desired characteristics. After going through five sections of helical pulse line, the ion beam has a mean velocity of around $9 \mathrm{~m} / \mu \mathrm{s}$ and velocity tilt $1 \mathrm{~m} / \mu \mathrm{s}$. The average kinetic energy of the ion beam at each helix junction is plotted in Fig. 11. The final average energy of the ion beam is around $2.8 \mathrm{MeV}$ after five sections of helix, which meets the ion beam requirement for NDCX-II. However, there are a few stray particles (less than $2 \%$ of the total charge) which turn back from the beam head. This small population has a negligible effect on the bulk of the beam and will likely be lost in the final focus section. The cause of the stray particles is probably due to the imperfect space charge cancellation from nonideal longitudinal beam profile. We see from the current evolution in Fig. 10 and the spatial distribution shown in Fig. 12 that the profile is quite nonparabolic. The transverse dynamics of the ion beam was also simulated for the design. The ion beam was able to be confined in the pipe and snapshots of the ion beam at
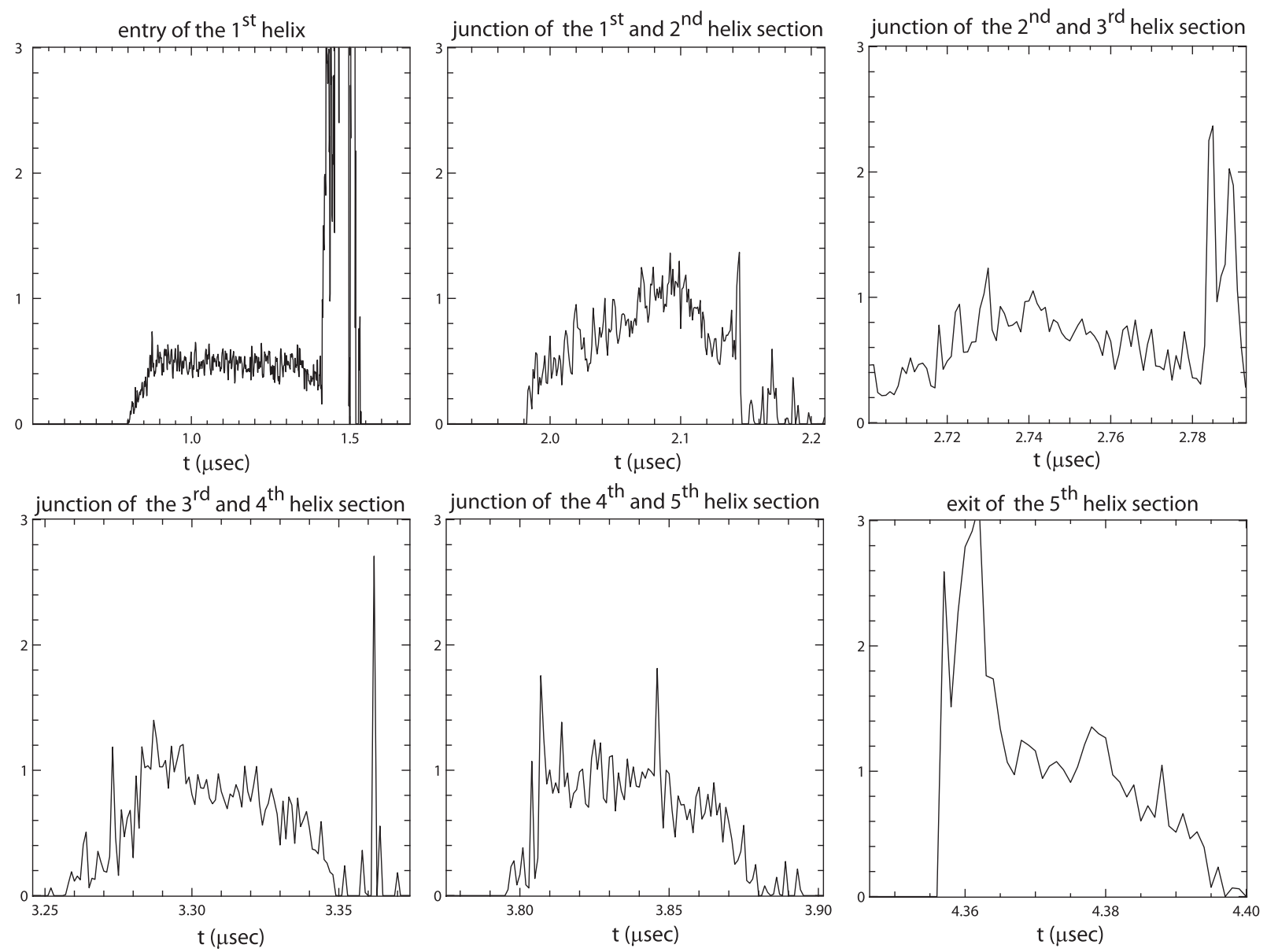

FIG. 13. The normalized emittance $\varepsilon_{n x}$ (in $\pi \mathrm{mm} \mathrm{mrad}$ ) of the ion beam versus time at various positions outside the helices. 

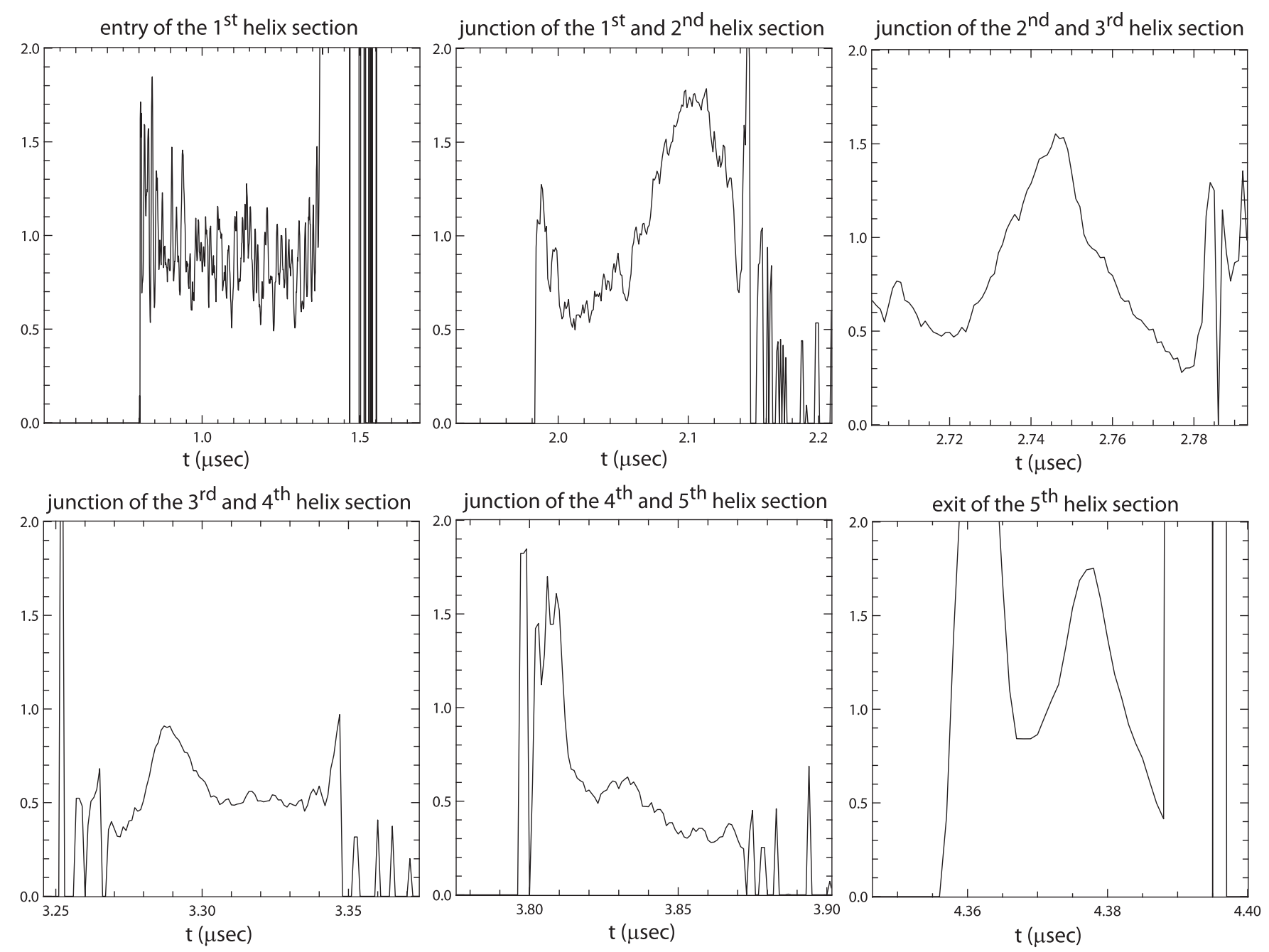

FIG. 14. The $\Delta p / p$ (in $10^{-3}$ ) of the ion beam versus time at varies positions outside the helices.

different section of helix are demonstrated in Fig. 12 again. The positions of the solenoids and their fields on axis are shown in Table II. Figure 13 shows the evolution of the normalized emittance $\varepsilon_{n x}$ of the ion beam. $\varepsilon_{n x}$ can be maintained to be around $1 \pi \mathrm{mm} \mathrm{mrad}$, except at the exit of the fifth section, where it grows to around $3 \pi \mathrm{mm} \mathrm{mrad}$ due to the distortion of phase space of the ion beam head mentioned before. The evolution of $\Delta p / p$ is shown in Fig. 14. This diagnostic only included the velocity of the ion beam particles within the range of $\bar{v}-3 \Delta v$ and $\bar{v}+$ $3 \Delta v$ for each slice, which is a fair measurement since the focusing section at a later stage would eliminate those outof-range ion beam particles. Under this scheme, $\Delta p / p$ of the main part of the ion beam can be kept under $2 \times 10^{-3}$. Stray particles do not affect the emittance and current diagnostics significantly. The final ion beam parameters after the five helix sections are shown in Fig. 15 and the longitudinal design is shown in Fig. 16.

Simulation results show that an ion beam with mean energy $2.8 \mathrm{MeV}, \Delta p / p$ under $2 \times 10^{-3}$, normalized emit- tance $\varepsilon_{n}$ around $1.5 \pi \mathrm{mm}$ mrad and total charge $38 \mathrm{nC}$ was achieved.

\section{CONCLUSION}

We have presented results for the NDCX-II design from source to accelerator exit. The PLIA architecture provides a natural mechanism for controlling longitudinal beam dynamics, as we have shown. We have derived the normalized emittance $\varepsilon_{n x}$ and $\Delta p / p$ at accelerator exit using the particle distribution with these characteristics whose growths are acceptable; we plan as our next step to propagate the ion beam through drift compression and final focus onto the target. The whole acceleration is $17 \mathrm{~m}$ long and indeed PLIA in the snowplow mode is not a high gradient device. However, since the structure is relatively simple, whether the cost is competitive in the long run must await detailed engineering designs. An electrical flashover problem [5] on the PLIA insulator observed at LBNL has to date deterred progress towards a full experimental cam- 

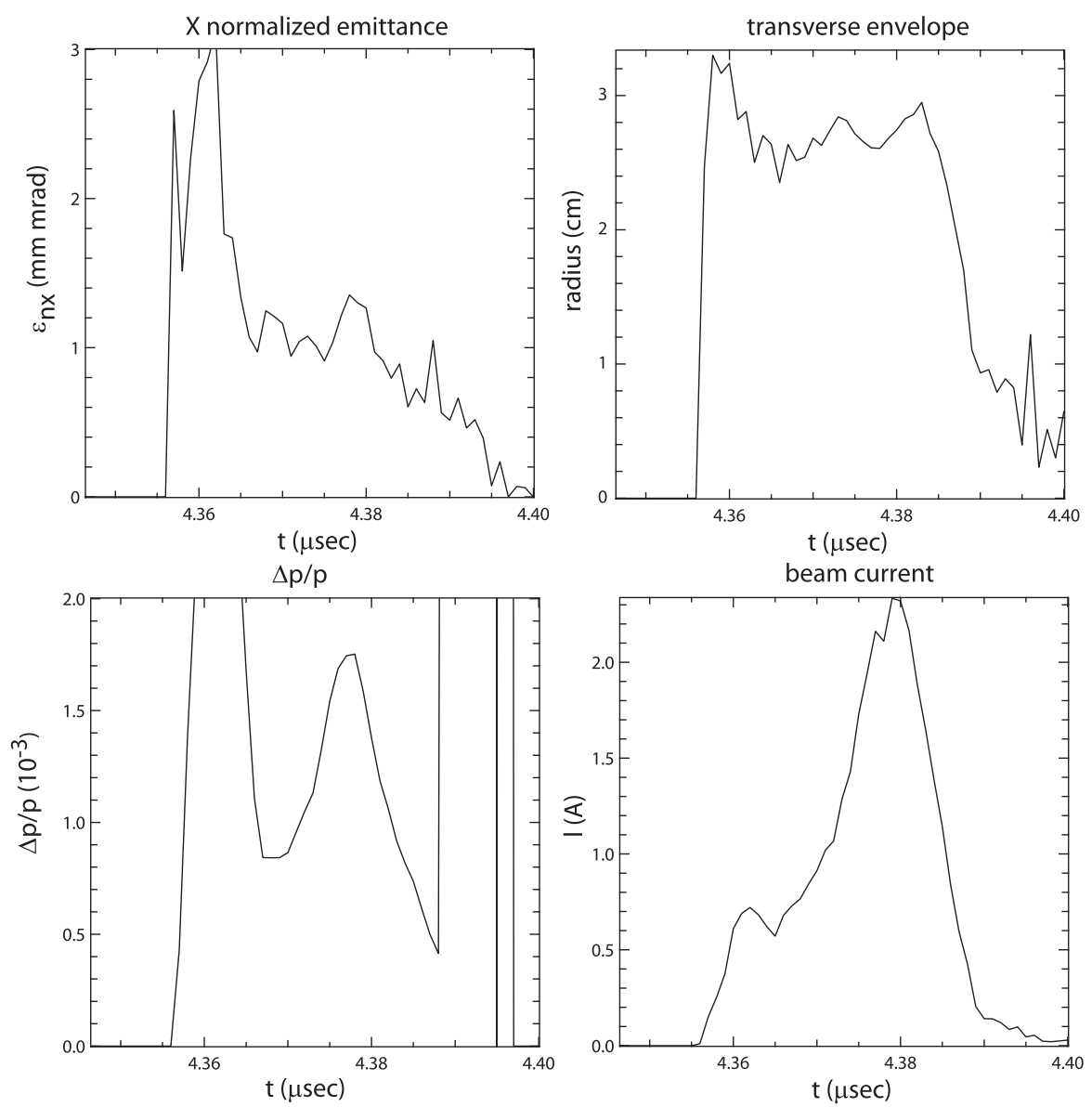

FIG. 15. Key moments of the ion beam as functions of time, measured at $z=17.5 \mathrm{~m}$, which is just before the entrance of induction for voltage tailoring.

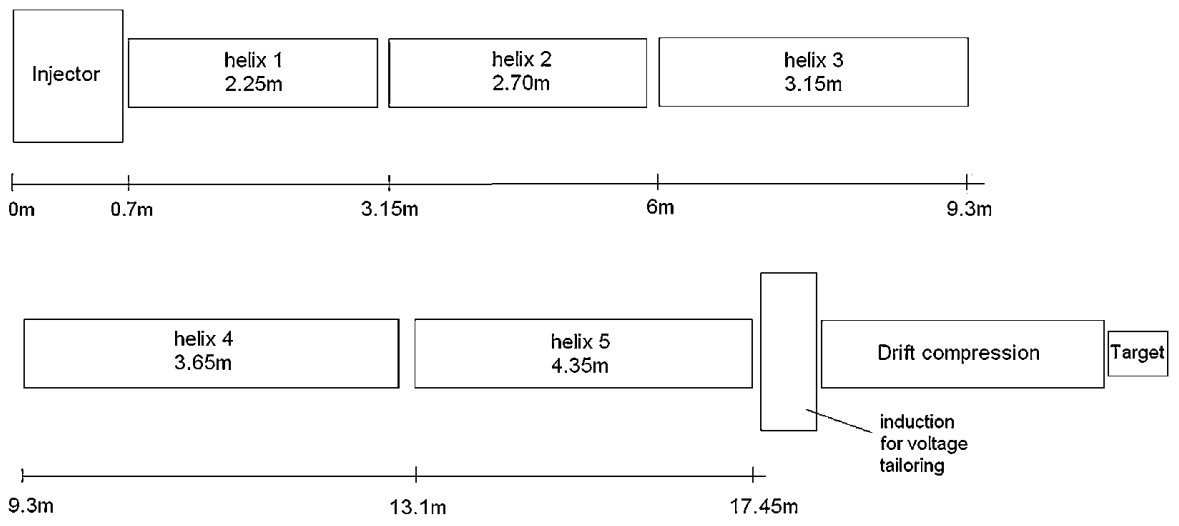

FIG. 16. The longitudinal design for PLIA-based NDCX-II.

paign. However, a previous paper [12] suggests that suppression of spurious high-frequency modes may eliminate this flashover.

\section{ACKNOWLEDGMENTS}

We would like to thank Will Waldron for suggesting the technical parameters of the helix structure used in these studies. 
[1] R. J. Briggs, Phys. Rev. ST Accel. Beams 9, 060401 (2006).

[2] J. E. Coleman et al., Nucl. Instrum. Methods Phys. Res., Sect. A 577, 197 (2007).

[3] A. Friedman et al., in Proceedings of the 21st Particle Accelerator Conference, Knoxville, 2005 (IEEE, Piscataway, NJ, 2005), p. 339.

[4] P. K. Roy et al., Phys. Rev. ST Accel. Beams 9, 070402 (2006).

[5] W. L. Waldron et al., in Proceedings of the 2007 Particle Accelerator Conference, Albuquerque, New Mexico, 2007 (IEEE, Albuquerque, New Mexico, 2007), p. 852.
[6] A. Friedman et al., in Proceedings of the 17th International Symposium on Heavy Ion Inertial Fusion (2008).

[7] J. J. Barnard et al. (Ref. [6]).

[8] P. K. Roy et al., Phys. Rev. Lett. 95, 234801 (2005).

[9] J.J. Barnard et al., in Proceedings of the 21st Particle Accelerator Conference, Knoxville, 2005 (Ref. [3]), p. 2568.

[10] D. P. Grote et al., AIP Conf. Proc. 749, 55 (2005).

[11] D. A. Callahan et al., AIP Conf. Proc. 297, 211 (1993).

[12] C. Y. Ling et al. (Ref. [6]). 